\title{
Avaliação de barreiros e finalidade da água armazenada na região semi-árida da Bahia
}

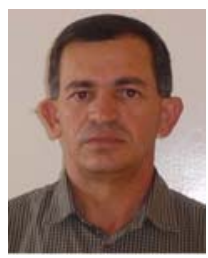

Nilton de B. Cavalcanti ${ }^{1}$ \& Geraldo M. Resende ${ }^{2}$

\author{
1 Embrapa Semi-Árido, CP 23. CEP 56.304-408, Petrolina, PE. Fone: (81) 3862-1711. E-mail:nbrito@cpatsa.embrapa.br (Foto) \\ 2 Embrapa Semi-Árido, Fone: (81) 3862-1711. E-mail:gmilanez@cpatsa.embrapa.br
}

Protocolo $041-29 / 3 / 2001$

\begin{abstract}
Resumo: Fazer-se um levantamento do tipo e da quantidade de barreiros utilizados pelos pequenos agricultores das comunidades de Curaçá, Casa Nova e Jaguarari, municípios do Estado da Bahia, foi a meta principal deste trabalho, realizado no período de janeiro de 1998 a dezembro de 1999, em que os resultados obtidos demonstraram que os pequenos agricultores utilizam algum tipo de barreiro para armazenar água de chuva. A água é utilizada para o consumo humano e animal.
\end{abstract}

Palavras-chave: escoamento, barreiro, pequenos agricultores

\section{Evaluation of the water traps and stored water usage in the semi-arid region of Bahia - Brazil}

\begin{abstract}
This study had the objective of making a survey about the type and amount of water traps used as rainwater catchment by small farmers of three communities located in the municipalities of Curaçá, Casa Nova and Jaguarari in the State of Bahia, Brazil. The research was carried out between January of 1998 and December of 1999. The results showed that small farmers use some form of tank for rainwater catchment. The water is used for human and animal consumption.
\end{abstract}

Key words: rainwater, tank, small farmers

\section{INTRODUÇÃO}

Na região semi-árida do Nordeste brasileiro, a quantidade de água de chuva, segundo Rebouças \& Marinho (1972) é de aproximadamente 700 bilhões de $\mathrm{m}^{3} \mathrm{ano}^{-1}$, o que torna o semiárido nordestino diferente das demais regiões semi-áridas do mundo. A maior parte dessa chuva não é aproveitada em todo o seu potencial pois, mesmo existindo grande quantidade de barreiros e açudes no Nordeste, 36 bilhões de $\mathrm{m}^{3}$ se perdem pelo escoamento superficial. Segundo Silva et al. (1981) a maioria dos pequenos barreiros existentes no Nordeste não é utilizada para irrigação de salvação, porém já existe um tipo de barreiro adaptado pela pesquisa agrícola, que possibilita a captação e o armazenamento de água das chuvas que escoa no solo para utilizá-la nos períodos de estiagem, através da irrigação de salvação; no entanto, Cavalcanti et al. (1999) constataram que essa alternativa tecnológica para captação, armazenamento e utilização de água de chuva não é utilizada pelos agricultores do semi-árido nordestino. Segundo Lopéz (1999) a pesquisa agrícola é imprescindível ao propósito de se gerar tecnologia que sirva de base para propor formas de aumentar a rentabilidade dos produtores e, ao mesmo tempo, proporcionar o uso racional, conservação e recuperação dos recursos naturais. Souza (1999) afirma que, com prognóstico de períodos de seca e se conhecendo a variação temporal e espacial das precipitações, é de suma importância o fornecimento de subsídios ao planejamento da época de plantio e melhor adequação ao uso da irrigação. Neste contexto, o êxito da agricultura sustentável em regiões com escassez de recursos naturais está, segundo Paz et al. (2000), no desenvolvimento de metodologias e instrumentos tecnológicos apropriados a cada situação e região.

Por outro lado, os pequenos agricultores desta região enfrentam, a cada ano, problemas para suprirem as necessidades de água de seus animais e de sua família, porém, são poucos os que utilizam barragens e/ou barreiros para o armazenamento dessas águas. A pouca utilização dos barreiros se dá principalmente pelo custo de implantação, visto que, na maioria dos casos, a utilização de máquinas para sua construção, torna esta prática inviável para os pequenos agricultores. Os custos de implantação de um barreiro para irrigação de salvação, explorando uma área de 1,5 ha, com milho e feijão, estão na ordem de US\$ 2,200.00 (Brito et al., 1999). Segundo Pinheiro (1999) um dos principais obstáculos ao uso de barreiros para irrigação de salvação, é que, quando submetidos a longos períodos de estiagem, os pequenos agricultores preferem, então, guardar a água para o consumo doméstico e animal, que utilizá-la para irrigação. Este trabalho teve como objetivo fazer um 
levantamento dos tipos e da quantidade de barreiros utilizados pelos agricultores de três comunidades localizadas nos municípios de Curaçá, Casa Nova e Jaguarari, no Estado da Bahia, no período de janeiro de 1998 a dezembro de 1999.

\section{MATERIAL E MÉTODOS}

Neste estudo, selecionaram-se, ao acaso, três comunidades de pequenos agricultores nos municípios de Curaçá, Casa Nova e Jaguarari, no Estado da Bahia, sendo uma comunidade em cada município. Estes municípios foram selecionados entre municípios de Uauá, Juazeiro, Remanso, Canudos, Monte Santo e Queimadas, em função da severidade da seca ocorrida no período de janeiro de 1998 a dezembro de 1999. A região dos municípios é caracterizada como "Depressão Sertaneja" de clima muito quente, semi-árido, com chuvas no período de novembro a abril, cuja precipitação média anual é de $577,8 \mathrm{~mm}$ (Silva et al., 1993). Nesta região, a instabilidade climática é caracterizada mais pela irregularidade de distribuição da chuva que por sua escassez (Silva et al., 1993). Em cada comunidade foi realizado um levantamento por meio de um questionário aplicado junto aos agricultores de cada família, procurando identificar-se quantos barreiros havia na propriedade da família, a forma de construção e de utilização da água. O tamanho médio das propriedades nas comunidades selecionadas é de 35,8 ha. O trabalho foi realizado no período de janeiro de 1998 a dezembro de 1999. As variáveis analisadas foram as seguintes: 1) quantidade de barreiros na comunidade; 2) tipo do barreiro; 3) forma de utilização da água; 4) custos de construção, e 5) agricultores que conhecem o barreiro para irrigação de salvação. Os dados obtidos foram submetidos a análise estatística e utilizado o procedimento proc tabulate, que compõe o SAS (SAS Institute, 1990).

\section{RESULTADOS E DISCUSSÃO}

Pode-se observar, na Tabela 1, que nas comunidades analisadas a quantidade de barreiros é muito pequena, considerando-se o número de famílias residentes. Na comunidade de Fazenda Brandão (Curaçá, BA) das 17 famílias pesquisadas apenas 8 possuem barreiros, 6 barreiros foram construídos com mão-de-obra dos agricultores e seus familiares, nos anos de 1997 a 1998, e 2 barreiros foram construídos usando-se máquina cedida pela Prefeitura, com as despesas de combustíveis pagas pelos agricultores. A água armazenada é utilizada principalmente para o consumo animal e, eventualmente, para uso doméstico (Figuras 1 e 2). Essa forma de utilização da água dos barreiros, também foi observada por Silva et al. (1981).

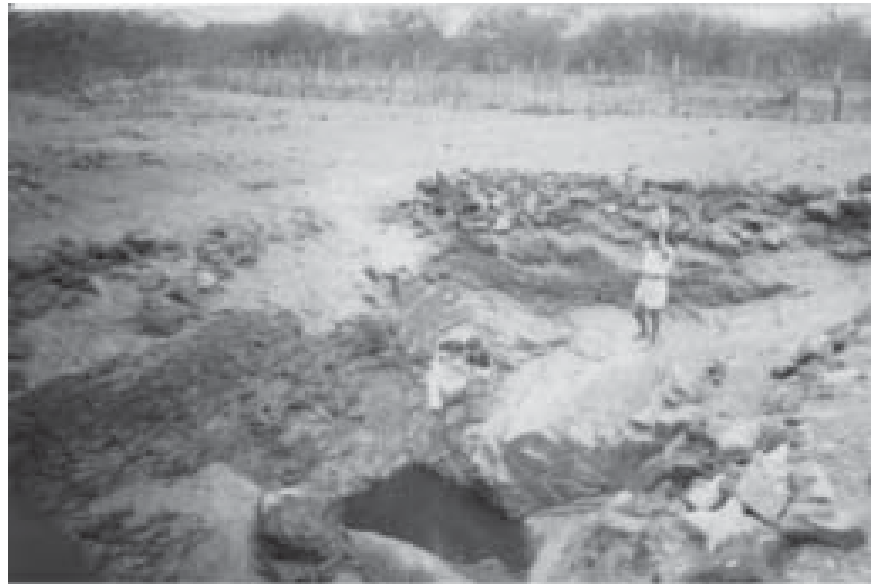

Figura 1. Barreiro com água para consumo humano

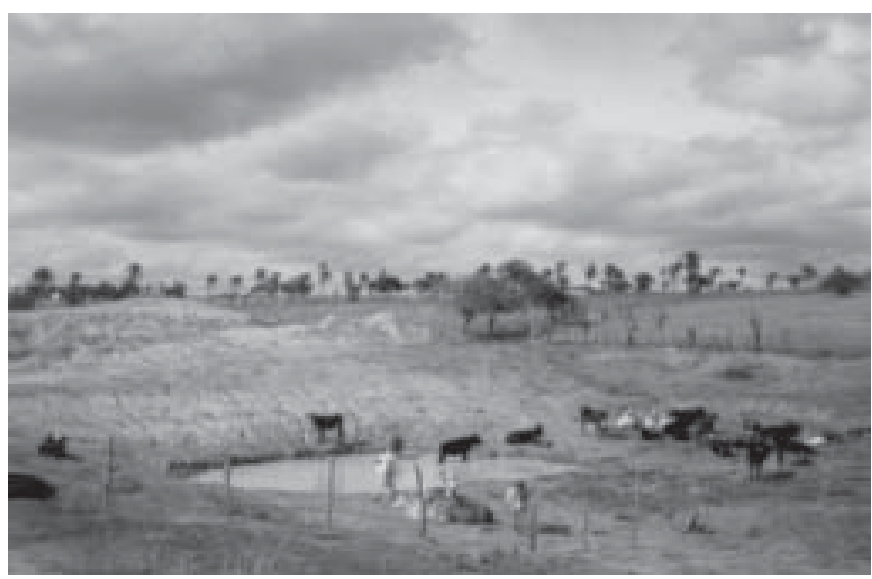

Figura 2. Barreiro com água para consumo animal

Nessa comunidade, $60 \%$ dos agricultores conhecem e/ou já ouviram falar do barreiro para irrigação de salvação (Figura 3) e $36 \%$ não utilizam esse tipo de barreiro, por falta de recursos financeiros, pois os custos de implantação de um barreiro para irrigação de salvação estão relativamente muito acima da disponibilidade financeira dos pequenos agricultores, conforme os orçamentos demonstrados por Brito et al. (1999) porém, segundo Pinheiro (1999) os obstáculos ao uso de barreiros para irrigação de salvação, é que, na estiagem, os agricultores preferem armazenar a água para o consumo doméstico e animal, que utilizá-la para irrigação.

Embora os recursos financeiros dos pequenos agricultores sejam escassos, o barreiro para irrigação de salvação é uma alternativa tecnológica de grande importância para a região semi-árida e sua concepção corrobora com a afirmação de Lopéz (1999) de que a pesquisa agrícola é imprescindível ao propósito de se gerar tecnologia que sirva de base para propor formas de aumentar a rentabilidade dos produtores e, ao mesmo tempo, proporcionar o uso racional, conservação e recuperação dos recursos naturais.

Tabela 1. Quantidade de barreiros nas comunidades, forma de utilização da água e período de armazenamento da água

\begin{tabular}{lccccc} 
Comunidade & $\begin{array}{c}\text { Número de } \\
\text { Famílias } \\
\left(\mathrm{n}^{1}\right)\end{array}$ & $\begin{array}{c}\text { Quantidade } \\
\text { de Barreiros }\end{array}$ & $\begin{array}{c}\text { Capacidade Média } \\
\text { dos Barreiros } \\
\left(\mathrm{m}^{3}\right)\end{array}$ & $\begin{array}{c}\text { Forma de Utilização } \\
\text { da Água }\end{array}$ & $\begin{array}{c}\text { Período médio de } \\
\text { Armazenamento } \\
\text { da Água }\end{array}$ \\
\hline Fazenda Brandão & 17 & 8 & 2.187 & Consumo humano e animal & 6 meses \\
Fazenda Saco & 33 & 12 & 3.215 & Consumo humano e animal & 8 meses \\
Sítio São Pedro & 23 & 9 & 2.924 & Consumo humano e animal & 7 meses \\
\hline
\end{tabular}




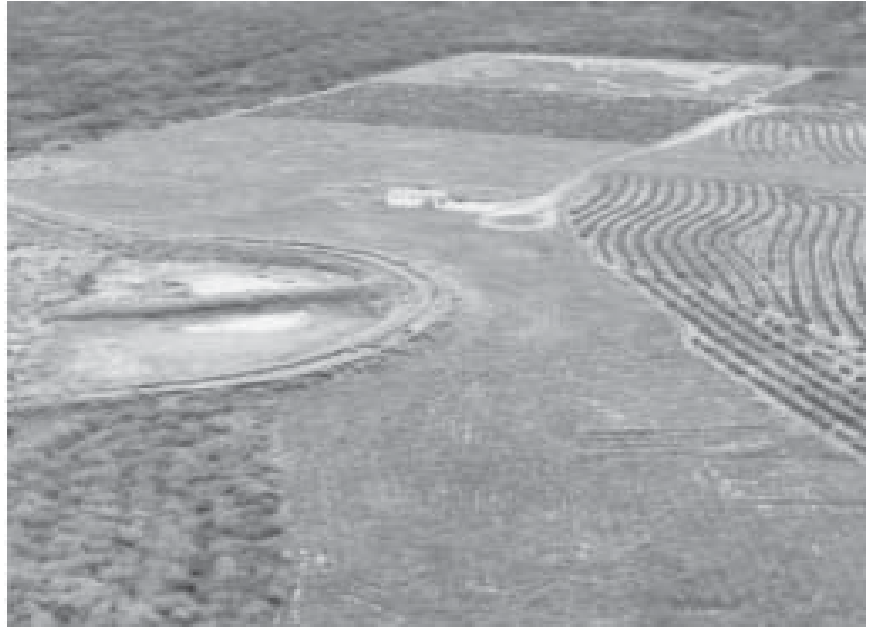

Figura 3. Barreiro para irrigação de salvação

Segundo Souza (1999) com prognóstico de períodos de seca e se conhecendo a variação temporal e espacial das precipitações, é de suma importância o fornecimento de subsídios ao planejamento da época de plantio e melhor adequação ao uso da irrigação. Neste contexto, alternativas inovadoras, como o barreiro para irrigação de salvação, são de grande valor para a região semi-árida nordestina.

A não utilização do barreiro para irrigação de salvação também foi constatada por Cavalcanti et al. (1999) em outros municípios do Nordeste.

Conforme Paz et al. (2000) para o desenvolvimento de uma região, há necessidade de tecnologias, as quais devem ser acessíveis e possíveis de serem adotadas pelos produtores, promovendo o aumento de produtividade, com o mínimo risco ao meio ambiente. Neste contexto, tecnologias alternativas, como barragem subterrânea, barreiros para irrigação de salvação e cisternas rurais, são imprescindíveis para o êxito dos pequenos agricultores no Nordeste semi-árido.

Na comunidade da Fazenda Saco (Jaguarari, BA) das 33 famílias apenas 12 possuem barreiros, cuja água é utilizada para o consumo humano e animal. Todos os barreiros dessa comunidade foram construídos pelos agricultores, nos anos de 1992 a 1995, e nenhum agricultor conhece o barreiro para irrigação de salvação. Na comunidade de Sítio São Pedro (Casa Nova, BA) foram pesquisadas 23 famílias, das quais 9 possuem barreiros que são utilizados para suprir as necessidades de água para os animais e para uso doméstico em período de seca. Todos os barreiros dessa comunidade foram construídos com mão-de-obra dos agricultores.

A maior parte dos barreiros encontrados nas comunidades é de forma circular e triangular, com profundidade de 3 a $4 \mathrm{~m}$, acumulando água por um período médio de 6 a 8 meses, em anos de chuvas regulares. A forma dos barreiros, na maioria dos casos, impede um acúmulo maior de água, corroborando com as afirmações de Rebouças \& Marinho (1972) de que a maior parte das chuvas que ocorrem na região Nordeste, é mal aproveitada.

\section{CONCLUSÕES}

Constatou-se um pequeno número de barreiros nas comunidades trabalhadas, os quais foram construídos sem planejamento do volume necessário para o atendimento da demanda de água das famílias rurais em períodos de seca. A maioria dos pequenos produtores desconhece a tecnologia do barreiro de salvação.

\section{LITERATURA CITADA}

Brito, L.T.L.; Porto, E.R.; Anjos, J.B. Barreiro para uso em irrigação de salvação. In: Simpósio sobre Captação de Água de Chuva no Semi-Árido Brasileiro, 1, 1997, Petrolina. Anais... Petrolina: Embrapa Semi-Árido/IRPAA/IRCSA, 1999. 186p.

Cavalcanti, N.B.; Oliveira, C.A.V.; Brito, L.T.L.; Resende, G.M. Avaliação do uso de técnicas de captação de água de chuva na região semi-árida do Nordeste brasileiro. Revista Brasileira Engenharia Agrícola e Ambiental, Campina Grande, v.3, n.3, p.403-407, 1999 .

Lopéz, J.K. El papel de la investigación agrícola en el combate a la probreza y conservación de los recursos naturales. Elementos para su decisión. Rede Internacional de Metodologia de Investigación de Sistemas de Producción. www.rimisp.cl/r/kondo.htm.1999.

Paz, V.P.S.; Teodoro, R.E.F.; Mendonça, F.C. Recursos hídricos, agricultura irrigada e meio ambiente. Revista Brasileira Engenharia Agrícola e Ambiental, Campina Grande, v.4, n.3, p.465-473, 2000 .

Pinheiro, R.N. Técnicas de captação de água de chuva: experiências do Rio Grande do Norte. In: Simpósio sobre Captação de Água de Chuva no Semi-Árido Brasileiro, 1., 1997, Petrolina. Anais... Petrolina: Embrapa Semi-Árido/ IRPAA/IRCSA, 1999. 186p.

Rebouças, A.C.; Marinho, M.E. Hidrologia das secas do Nordeste do Brasil. Recife, PE, SUDENE - DRN, Divisão de Hidrologia, 1972. 126p. Brasil. SUDENE. Hidrologia, 40

SAS INSTITUTE, SAS Language guide for personal, computers, release 6. 2.ed. Cary, NC. SAS Institute Inc., 1990. 319p.

Silva, A.S.; Porto, E.R.; Gomes, P.C.F. Seleção de áreas e construção de barreiros para uso em irrigação de salvação no trópico semi-árido. Petrolina, PE: Embrapa-CPATSA, 1981. 43p. il. Embrapa-CPATSA. Circular Técnica, 3

Silva, F.B.R.; Riché, G.R.; Tonneau, J.P.; Souza Neto, N.C.; Brito, L.T.L.; Correia, R.C.; Cavalcanti, A.C.; Silva, F.H.B.B.; Silva, A.B.; Araújo Filho, J.C. Zoneamento agroecológico do Nordeste: diagnóstico do quadro natural e agrossocioeconômico. Petrolina, PE: EMBRAPA-CPATSA/ Recife: EMBRAPA-CNPS. Coordenadoria Regional Nordeste, 1993. v.2.382p.

Souza, S. A. V. Programa computacional para simulação da ocorrência de veranicos e queda de produção. Piracicaba: ESALQ/USP, 1999. 124p. Tese Doutorado 